\title{
An update: improvements in imaging perfluorocarbon-mounted plant leaves with implications for studies of plant pathology, physiology, development and cell biology
}

\author{
George R. Littlejohn ${ }^{1}{ }^{*}$, Jessica C. Mansfield ${ }^{2}$, Jacqueline T. Christmas ${ }^{3}$, Eleanor Witterick ${ }^{1}$, \\ Mark D. Fricker ${ }^{4}$, Murray R. Grant ${ }^{1}$, Nicholas Smirnoff ${ }^{1}$, Richard M. Everson ${ }^{3}$, Julian Moger ${ }^{2}$ and \\ John Love ${ }^{\text {* }}$
}

${ }^{1}$ Division of Plant and Microbial Sciences, School of Biosciences, University of Exeter, Exeter, UK

${ }^{2}$ School of Physics, University of Exeter, Exeter, UK

${ }^{3}$ Computer Science, University of Exeter, Exeter, UK

${ }^{4}$ Department of Plant Sciences, University of Oxford, Oxford, UK

Edited by:

Markus Schwarzländer, University of Bonn, Germany

\section{Reviewed by:}

Viktor Zarsky, Charles University, Czech Republic

Jin-Long Qiu, Institute of

Microbiology - Chinese Academy of Sciences, China

David C. Logan, Université d'Angers, France

\section{*Correspondence:}

George R. Littlejohn and John Love, Division of Plant and Microbial

Sciences, School of Biosciences, University of Exeter, Geoffrey Pope Building, Stocker Road, Exeter EX4 4QD, UK

e-mail:G.R.Littlejohn@exeter.ac.uk; j.love@exeter.ac.uk
Plant leaves are optically complex, which makes them difficult to image by light microscopy. Careful sample preparation is therefore required to enable researchers to maximize the information gained from advances in fluorescent protein labeling, cell dyes and innovations in microscope technologies and techniques. We have previously shown that mounting leaves in the non-toxic, non-fluorescent perfluorocarbon (PFC), perfluorodecalin (PFD) enhances the optical properties of the leaf with minimal impact on physiology. Here, we assess the use of the PFCs, PFD, and perfluoroperhydrophenanthrene (PP11) for in vivo plant leaf imaging using four advanced modes of microscopy: laser scanning confocal microscopy (LSCM), two-photon fluorescence microscopy, second harmonic generation microscopy, and stimulated Raman scattering (SRS) microscopy. For every mode of imaging tested, we observed an improved signal when leaves were mounted in PFD or in PP11, compared to mounting the samples in water. Using an image analysis technique based on autocorrelation to quantitatively assess LSCM image deterioration with depth, we show that PP11 outperformed PFD as a mounting medium by enabling the acquisition of clearer images deeper into the tissue. In addition, we show that SRS microscopy can be used to image PFCs directly in the mesophyll and thereby easily delimit the "negative space" within a leaf, which may have important implications for studies of leaf development. Direct comparison of on and off resonance SRS micrographs show that PFCs do not to form intracellular aggregates in live plants. We conclude that the application of PFCs as mounting media substantially increases advanced microscopy image quality of living mesophyll and leaf vascular bundle cells.

Keywords: perfluorocarbon, Arabidopsis, multi-photon, confocal, microscopy, imaging, perfluoroperhydrophenanthrene

\section{INTRODUCTION}

Advances in microscopy have made in vivo biological imaging increasingly important in recent years. A diverse palette of chemical labels and genetically encoded fluorescent reporters and biosensors, coupled with advanced microscopy techniques, including laser scanning confocal microscopy (LSCM), twophoton fluorescence (TPF) microscopy and label-free imaging techniques such as second harmonic generation (SHG) microscopy and stimulated Raman scattering (SRS) microscopy have enabled unprecedented analysis of living cell dynamics (Shaw and Ehrhardt, 2013). Despite these technical advances, tissues that are rich in airspaces, such as those of plant leaves or animal lungs, remain difficult to image because of the optical aberrations that result from the complex structure of such tissues. As these tissues are typically sites of active metabolism and often targets for pathogens, it is essential to develop relatively simple in vivo methods to circumvent these imaging problems.

The spongy mesophyll of higher plant leaves is located adjacent to the lower epidermis, contains numerous airspaces and may be several cell layers thick (Figure 1A). These characteristics result in light refraction within the mesophyll and a progressive attenuation of light transmission through the tissue, producing optical aberrations that impair confocal image quality (Feijó and Moreno, 2004; Inoue, 2006; Cheng, 2006). When examining fixed leaves these aberrations are minimized because the fixatives infiltrate the tissue and minimize the optical phase transitions within. Previously, we have shown that it is possible to infiltrate living leaves with perfluorodecalin (PFD, Figure 1B) and thereby significantly improve the resolution of LCSM images of the mesophyll while affecting only minimally cellular physiology (Littlejohn et al., 2010). We 

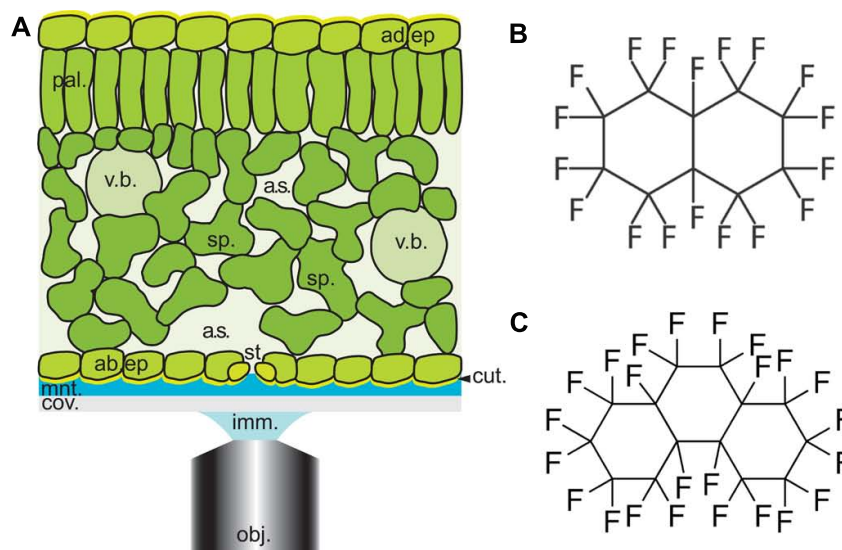

C

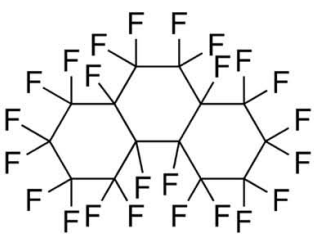



FIGURE 1 | Arabidopsis leaf anatomy, chemical structures of perfluorocarbons used in this study and a typical image of Pseudomonas infected leaves. (A) Diagrammatical representation showing the anatomical features of the Arabidopsis leaf in relation to the optical set-up. Abbreviations used are obj, objective lens; imm, immersion fluid; cov, coverslip; mnt, mountant; cut, cuticle; ad. ep, adaxial epidermis; st, stomatal pore; sp, spongy mesophyll; a.s, airspace; pal, palisade mesophyll; v.b, vascular bundle; ad. ep, adaxial epidermis. Cell walls are indicated by black lines (reproduced with permission from Littlejohn and Love, 2012). (B,C) chemical structures of (A) PFD and (B) PP11. (D) Z-stack reconstruction of GFP-expressing Pseudomonas syringae pv. tomato DC3000 infected Arabidopsis leaf. GFP signal is shown in green and chlorophyll autofluorescence in red. Scale bar is $25 \mu \mathrm{m}$. have had numerous positive reports from users of PFD and some studies have now been published (Johnson et al., 2011; Knapp et al., 2012; Tschiersch et al., 2012; Carrión et al., 2013; Gest et al., 2013; Hoepflinger et al., 2013; Hutt et al., 2013; Mansfield et al., 2013; Wright et al., 2013).

In this paper, we report that the optical qualities of plant mesophyll can be further improved by infiltration with perfluoroperhydrophenanthrene (PP11, Figure 1C), a perfluorocarbon (PFC) that has a refractive index that is better matched to that of living cells. To measure the improvement in image quality more objectively, we developed a method of autocorrelation that quantifies the sharpness of the images acquired at varying depths within the leaf. Finally, using SRS microscopy we have shown that both PFD and PP11 are undetectable in living cells, but infiltrate the mesophyll airspaces homogenously. Furthermore, SRS imaging of PFCs in vivo may be used to delimit the "negative space" within plant leaves, i.e., the area within the leaf that is involved in gaseous exchange and pathogen invasion. We have advocated the application of PFC mounting of samples to studies of pathogenesis in plants and experiments to date are promising. For example, Figure 1D shows an example image of an Arabidopsis thaliana leaf infected with the pathogenic bacterium, Pseudomonas syringae pv. tomato (DC3000 expressing GFP), taken under the same conditions as those used in Hutt et al. (2013).

\section{MATERIALS AND METHODS PLANT CULTURE AND SAMPLE MOUNTING}

Arabidopsis thaliana (Col-0 ecotype) and transformants that stably and constitutively express a cytoplasmically localized "Venus" yellow fluorescent protein (SEYFP-F46L; Nagai et al., 2002) were used in this study. Seeds were surface sterilized for 3 min with $70 \%$ ethanol and then for 5 min with $10 \%$ sodium hypochlorite. Seeds were washed five times in water and suspended in $0.1 \%$ agar. Seeds were stratified at $4^{\circ} \mathrm{C}$, in the dark, for $48 \mathrm{~h}$ before being sown on compost and grown at $20^{\circ} \mathrm{C}$, in a $16 \mathrm{~h} / 8 \mathrm{~h}$ light/dark photoperiod.

Mature leaves were excised from plants aged approximately 3-weeks and sections floated in $\mathrm{H}_{2} \mathrm{O}$, PFD, or PP11 for $5 \mathrm{~min}$ according to the methods described in Littlejohn et al. (2010), Littlejohn and Love (2012). Samples were mounted in the same medium and imaged by LSCM, TPF, SHG, and SRS microscopy.

\section{LSCM IMAGING}

Confocal imaging was performed using a Zeiss Axiovert 510 Meta LSCM equipped with a $40 \mathrm{x} / 1.30$ oil DIC immersion C-Apochromat lens. Immersion medium was Zeiss immersol. Light paths and wavelengths were controlled by a $458 / 514 \mathrm{~nm}$ dichroic mirror. The pinhole was set at $70 \mu \mathrm{m}$. Images were integrated and processed using Zeiss 510 software. Images of Venus and chlorophyll fluorescence in intact Arabidopsis leaves were collected with excitation at $514 \mathrm{~nm}$ using a $30 \mathrm{~mW}$ argon laser, $6.1 \mathrm{~A}, 21.8 \%$ transmission intensity. Emission was recorded at 518-604 $\mathrm{nm}$ for Venus and at $647-690 \mathrm{~nm}$ for chlorophyll. Z-stacks containing $100 \mathrm{z}$-planes taken with $1 \mu \mathrm{m}$ step size were collected for each of five samples incubated in PFD, PP11, or $\mathrm{H}_{2} \mathrm{O}$ for 5 min before imaging and mounted in the same medium for imaging. Figures were assembled in Adobe Indesign. Figure 2 LSCM images were generated by using the "cut" function in Zeiss LSM Image Browser software, where the plane presented represents a cut through the entire $\mathrm{z}$-stack from top to bottom taken at an angle of $-24^{\circ}$. The images therefore show a progression through the stack from top to bottom. Single z-planes are presented in Figure 3. GFP-expressing Pseudomonas (made according to Lambertsen etal., 2004) and chlorophyll shown in Figure 1D were excited respectively with 488 and $633 \mathrm{~nm}$ laser lines and emission captured from 505 to $570 \mathrm{~nm}$ (GFP) 


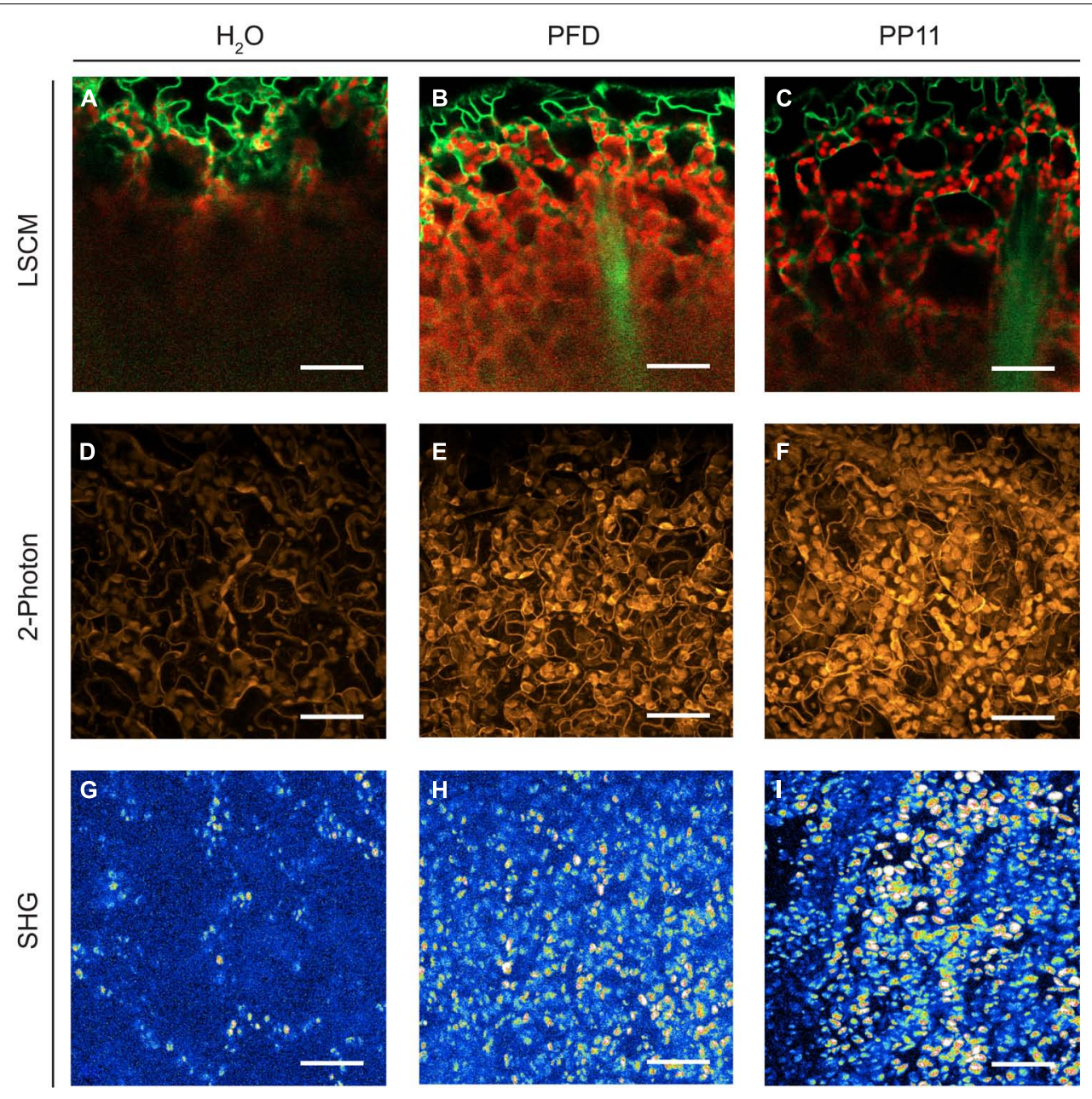

FIGURE 2 | Perfluorocarbon mounting media used with three modes of microscopy. (A-C) LSCM images captured from $1 \mu \mathrm{m}$ resolution Z-stacks taken of samples mounted in $\mathrm{H}_{2} \mathrm{O}$, PFD and PP11 respectively. Images were generated by using the "cut" function in Zeiss LSM Image Browser software, where the plane presented represents a cut through the entire z-stack from top to bottom taken at an angle of $-24^{\circ}$. The images therefore show a progression through the stack from top to bottom. Signal was collected from 518 to $604 \mathrm{~nm}$ for Venus, and is displayed in green and at $647-690 \mathrm{~nm}$ for chlorophyll, shown in red. (D-F) 2-photon micrographs taken of samples mounted in $\mathrm{H}_{2} \mathrm{O}, \mathrm{PFD}$, and PP11 respectively. (G-I) SHG micrographs taken of samples mounted in $\mathrm{H}_{2} \mathrm{O}, \mathrm{PFD}$, and PP11 respectively. (D-I) are presented as projections of z-stacks. Scale bars are $50 \mu \mathrm{m}$. and 647-711 nm (chlorophyll). The GFP-Pseudomonas Z-stack projected in Figure 1D was $30 \mu \mathrm{m}$ deep, with a $0.25 \mu \mathrm{m}$ step size and was captured with a C-Apochromat $63 \mathrm{x} / 1.2 \mathrm{~W}$ Corr M27 lens. The projection was made in Zeiss LSM510 software.

\section{RAMAN SPECTROSCOPY}

Prior to SRS imaging the Raman spectra of the PFCs were obtained using a Renishaw RM100 Raman microscope (Renishaw plc, UK), with a $785 \mathrm{~nm}$ diode laser and 1200 line/mm spectral grating, giving a spectral resolution of $1 \mathrm{~cm}^{-1}$.

\section{STIMULATED RAMAN SCATTERING MICROSCOPY}

Stimulated Raman scattering microscopy required two, pulsed laser beams; one at a longer wavelength (Stokes beam) and the other at a shorter wavelength (pump beam). The difference between the wavelengths of the pulsed lasers was tuned to correspond to the energy of a Raman vibration of interest. In our system a $1064 \mathrm{~nm}$ pico-second laser (PicoTrain HighQ laser) provided the Stokes beam and the output from an optical parametrical oscillator OPO (Levante Emerald APE) - pumped by the frequency doubled output of the picoTrain laser - provided the pump beam. The pump beam was tuned to a wavelength of $991.4 \mathrm{~nm}$, which excited the C-F vibrations at $687.5 \mathrm{~cm}^{-1}$.

The amplitude of the Stokes beam was modulated at $1.7 \mathrm{MHz}$ using an EOM. When SRS occurred within the sample, a modulated decrease in pump beam intensity [stimulated Raman loss; Moger et al. (2012)] was detected.

A modified laser scanning confocal microscope (Fluoview 300 IX71 Olympus) was used for imaging the SRS. The objective was a 60x 1.2 NA water immersion objective (UPlanS Apo Olympus). The transmitted light from the sample was collected with a $60 \times 1.0$ NA water-dipping condenser and detected using a photo-diode 

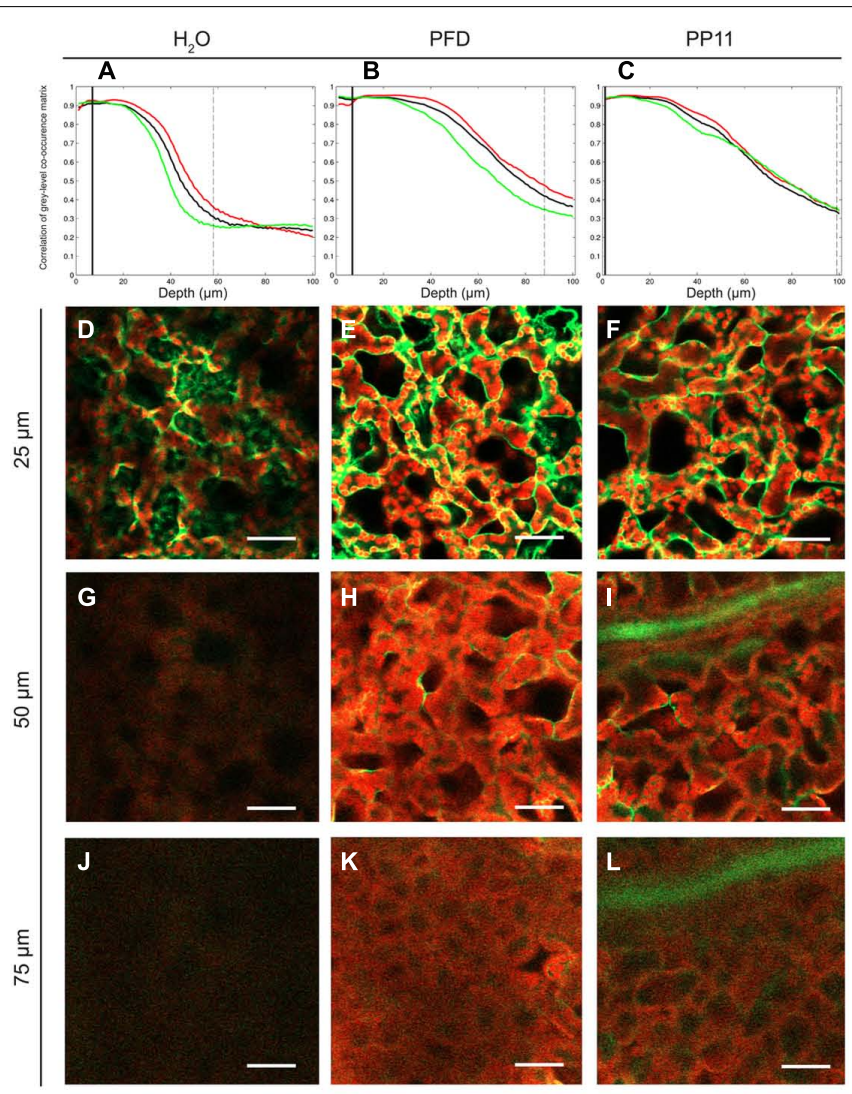

M

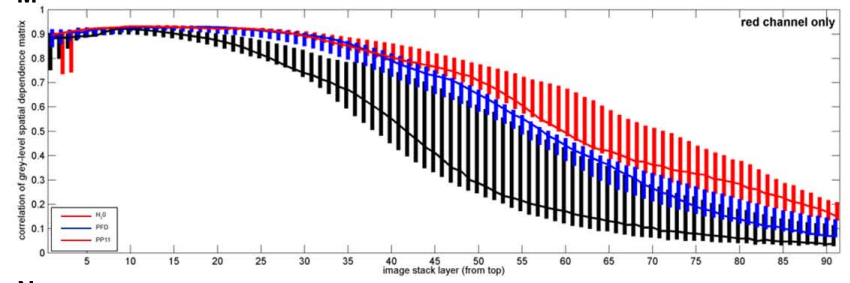

$\mathbf{N}$
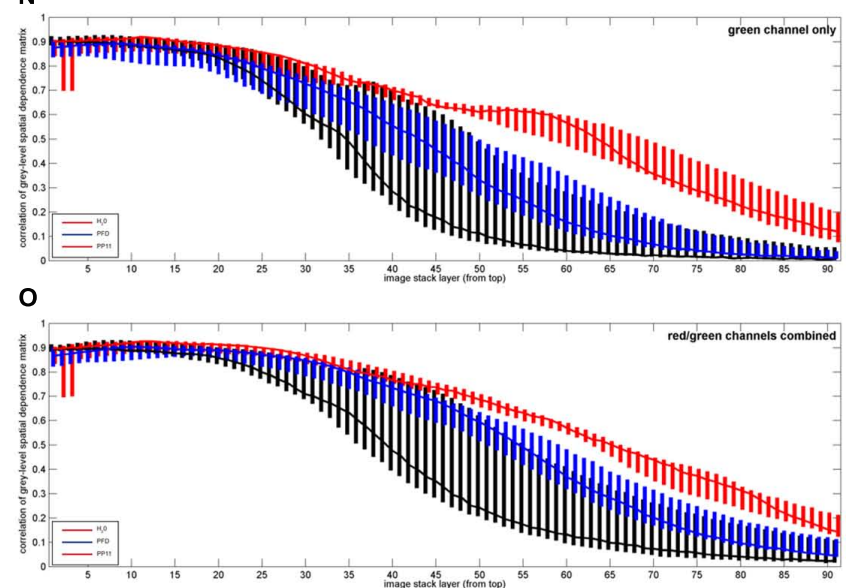

FIGURE 3 | Quantification of image deterioration with depth.

(A-C) typical autocorrelation results for single samples mounted in $\mathrm{H}_{2} \mathrm{O}$, PFD, and PP11 respectively and imaged as z-stacks $100 \mu \mathrm{m}$ deep. (D-F) representative images taken at a depth of $25 \mu \mathrm{m}$ from samples mounted in $\mathrm{H}_{2} \mathrm{O}, \mathrm{PFD}$, and PP11 respectively (G-I) representative images taken at a depth of $50 \mu \mathrm{m}$ from samples mounted in $\mathrm{H}_{2} \mathrm{O}$, PFD, and PP11 respectively. Signal was collected from 518 to $604 \mathrm{~nm}$ for Venus, and is displayed in green and at 647-690 nm for chlorophyll, shown in red. (J-L) representative images taken at a depth of $75 \mu \mathrm{m}$ from samples mounted in $\mathrm{H}_{2} \mathrm{O}$, PFD, and PP11 respectively. (M-O) range and median plotted for all samples ( $n=5)$ mounted in $\mathrm{H}_{2} \mathrm{O}$, PFD, or PP11 for red, green, and total signal respectively. Scale bars are $50 \mu \mathrm{m}$. 
with a $70 \mathrm{~V}$ reverse bias (FDS1010 thorlabs). The $1064 \mathrm{~nm}$ Stokes beam was blocked from reaching the photo-diode using a band pass filter coherent anti-Raman scattering (CARS; 890/220 nm, Chroma technologies). A lockin amplifier was used to separate out the modulated SRL signal and the output from this was connected to the computer and imaging software. Raman Image stacks were acquired at a $512 \times 512$ pixel resolution and a $256 \mu \mathrm{m} \times 256 \mu \mathrm{m}$ scan area with $1 \mu \mathrm{m}$ separation between optical slices.

\section{TWO-PHOTON FLUORESCENCE AND SECOND HARMONIC GENERATION MICROSCOPY}

Two-photon fluorescence and SHG were performed using the customized microscope described above. Excitation was provided by mode-locked femtosecond Ti:sapphire oscillator (Mira 900D; Coherent, USA) which produced $100-$ fs pulses at $76 \mathrm{MHz}$. The central wavelength of the fs beam was $800 \mathrm{~nm}$ with an average power at the sample of 5-30 mW. TPF and SHG were spectrally separated from the $800 \mathrm{~nm}$ excitation beam by a dichroic mirror (670dcxr; Chroma Technologies). After this, different bandpass filters were used to enable either TPF signal (CG-BG-39-1.00-1 and F70-500-3-PFU; CVI Melles Griot, UK) or SHG signal (F10-4005-QBL; CVI Melles Griot, UK) to reach the Hamamatsu R3896 photomultiplier tube.

\section{QUANTIFICATION OF IMAGE CLARITY IN THE Z-AXIS}

The clarity of each image in relation to its position in the $\mathrm{z}$-axis (i.e., into the tissue) was quantified using textural analysis. For each image, the "green" and "red" color channels were analyzed separately and in combination. A gray-scale spatial dependence matrix (Haralick et al., 1973), labeled "M," was constructed from the intensities (ranging from 0 to 255) of each pixel that composed each image and for each channel. The element $\mathrm{M}_{r, c}$, at row $r$ and column $c$ of this matrix is a count of how many times a pixel with intensity $r$ has a pixel of intensity $c$ in its immediate neighborhood, defined as the 8 pixels surrounding the measured pixel. Normalizing this matrix resulted in a set of joint probabilities of pixels with intensities $r$ and $c$ within the neighborhood. The correlation value associated with this normalized matrix (see Haralick et al., 1973) gives a measure of how closely correlated the intensity of a given pixel is with those of its neighboring pixels. The correlation measure was calculated for each image in acquired $\mathrm{z}$-stacks, $100 \mu \mathrm{m}$ deep, with a $1 \mu \mathrm{m}$ z-resolution for leaves mounted in $\mathrm{H}_{2} \mathrm{O}, \mathrm{PP} 11$ and PFD stacks, and separately for the red, green, and combined $\mathrm{red} /$ green channels.

\section{RESULTS \\ PERFLUOROCARBON MOUNTING MEDIA IMPROVE THE OPTICAL RESOLUTION OF DIFFERENT MODES OF LASER SCANNING MICROSCOPY}

We compared the image resolution of micrographic z-stacks acquired using LSCM, TPF, and SHG microscopy (Figure 2). TPF and SHG are non-linear optical techniques, which involve the simultaneous absorption of two or more photons. All these techniques are intrinsically confocal and generally use infra-red lasers instead of visible or UV lasers to excite fluorophores (Nandakumar et al., 2009). These techniques are considered advantageous compared to single-excitation confocal microscopy because they can deliver improved imaging depths within scattering tissues and reduced photodamage within samples.

Two-photon fluorescence is similar to single photon fluorescence, but it requires the simultaneous absorption of two near infra-red photons, rather than a single UV or visible spectrum photon, to generate a fluorescent signal (Diaspro and Sheppard, 2002).

Second harmonic generation involves the simultaneous absorption of two near infra-red photons and the emission of a single visible photon with half the wavelength of the infra-red photons. This process only occurs in structures which lack inversion symmetry. In plant tissues these structures include cellulose and starch grains (Mizutani et al., 2000; Brown et al., 2003; Cox et al., 2005).

Under all modes of microscopy tested, PP11 and PFD outperformed $\mathrm{H} 2 \mathrm{O}$ as an in vivo mounting medium, by infiltrating the mesophyll airspaces and smoothing the optical transitions within the mounted leaves. Moreover, we noted that PP11 outperformed PFD, which we ascribe to the refractive index of PP11 being closer to that of living cells compared to PFD. We were routinely able to acquire images from a depth of 100-135 $\mu \mathrm{m}$ within the leaf by LSCM and two photon imaging using PP11 as a mounting medium, which is greater than half the thickness of a leaf of a 3 week-old Arabidopsis plant and tests in with rice leaves allow imaging through the entire thickness of the leaf. The use of PFCs as a mounting medium also allowed the acquisition of SHG signals from chloroplast starch, which was not possible for samples mounted in $\mathrm{H} 2 \mathrm{O}$. This may, in itself, represent an important technique for the study of starch in leaves and statoliths in root cells.

To quantify the apparent advantage of using PFC mounting media to image within the mesophyll, we performed autocorrelation analysis on LSCM micrographs acquired at varying depths in Arabidopsis leaves. In this case, the fluorescence emission for both cytoplasmically localized Venus (Nagai et al., 2002) and chlorophyll were recorded. The pixel-by-pixel autocorrelation enabled an objective quantification of image quality. Using this method, we noted a wavelength-dependent improvement in resolution when samples were mounted in PFCs, compared to $\mathrm{H}_{2} \mathrm{O}$ (Figure 3). Images recorded deeper in the sample are more greatly affected by noise, which tends to be uncorrelated, and hence the correlation measure is low compared with the crisper images recorded closer to the surface. Median values for the autocorrelation demonstrate that PP11 and PFD outperform $\mathrm{H}_{2} \mathrm{O}$ and PP11 performs better than PFD, with a greater benefit seen in imaging Venus, compared with chlorophyll (Figures 3M-O). Most interestingly, the use of PFCs as mounting medium reduced the range of autocorrelation values obtained, showing that the images acquired when samples are mounted in PFD or in PP11 are not only clearer, but more consistent between replicate samples.

Normal cytoplasmic streaming and chloroplast movement was observed in all the experiments performed in this investigation, which is consistent with previous observations that the use of PFCs has a minimal effect on leaf physiology compared to mounting leaf samples in $\mathrm{H}_{2} \mathrm{O}$ (Littlejohn et al., 2010). This is also evidenced by the differences in chloroplast position seen between z-stacks represented in Figures 4G-J. 


\section{STIMULATED RAMAN SCATTERING IMAGING OF PERFLUOROCARBON DISTRIBUTION}

TPF and SHG and SRS are non-linear optical techniques, which involve the simultaneous absorption of two or more photons. All these techniques are intrinsically confocal and generally use infrared lasers instead of visible or UV lasers to excite fluorophores.

Stimulated Raman scattering is a chemical-specific technique which relies on stimulating Raman active molecular bond vibrations. SRS requires stimulation by two laser beams at different wavelengths (pump and Stokes beams), with the difference in wavelength between the two beams set to correspond to the energy of the molecular bond vibration of interest. When this condition is met, SRS occurs and results in a loss of intensity in the higher energy pump beam (stimulated Raman loss) and an equal increase in intensity in the Stokes beam (stimulated Raman gain). This process is detected by modulating one of the beams and detecting the modulations in intensity in the second beam using a lockin amplifier (Freudiger et al., 2008).

Perfluorocarbons readily infiltrate leaf airspaces. To ascertain whether PFCs remain localized in the airspaces of the mesophyll or are capable of also penetrating living cells, we imaged the PFC distribution in infiltrated Arabidopsis leaves using SRS. Prior to imaging, we demonstrated that Raman spectra may be used to identify specific peaks, which allow the C-F bonds in the PFC and the $\mathrm{C}-\mathrm{H}$ bonds found in Arabidopsis surface waxes, in cellulose and in $\beta$-1-3-glucan to be easily distinguished (Figures $4 \mathbf{A}-\mathbf{D}$ ). PFC mounting media are therefore compatible with Ramanbased imaging of biological molecules (Figures $4 \mathrm{E}, \mathrm{F}$ ). Images of the "ON" and "OFF resonance confirmed that PFCs was homogeneously distributed in the airspaces between mesophyll cells (Littlejohn etal., 2010). However, the sensitivity of this technique enables us also to confirm that PFCs do not appear to form intracellular aggregates, and are therefore unlikely to penetrate beyond the apoplast into the protoplasm (Figures $\mathbf{4 G - J}$ ). From a structural perspective, SRS imaging of PFC distribution in leaves has the added advantage of delimiting the mesophyll airspaces, potentially enabling a more intricate understanding and modeling of gas flow dynamics in leaves. The chloroplasts appear in both the on and off resonance SRS images due to their strong two-photon absorption (TPA). In this process they are absorbing one photon originating from the pump beam (either 991 or $989 \mathrm{~nm}$ on or off resonance) and one photon
A

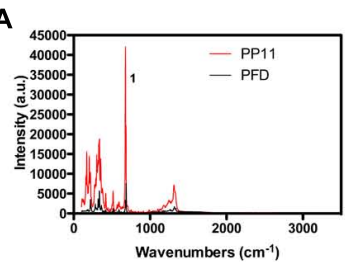

B

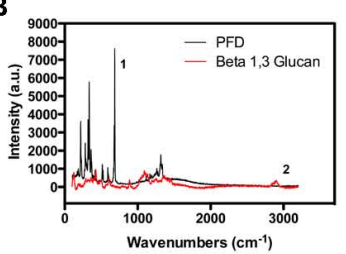

C

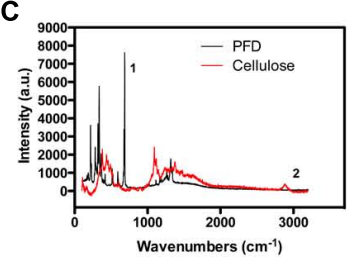

D

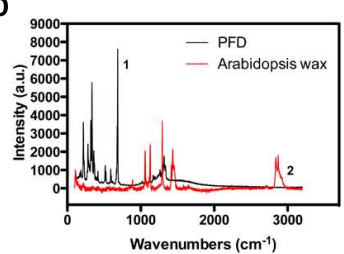

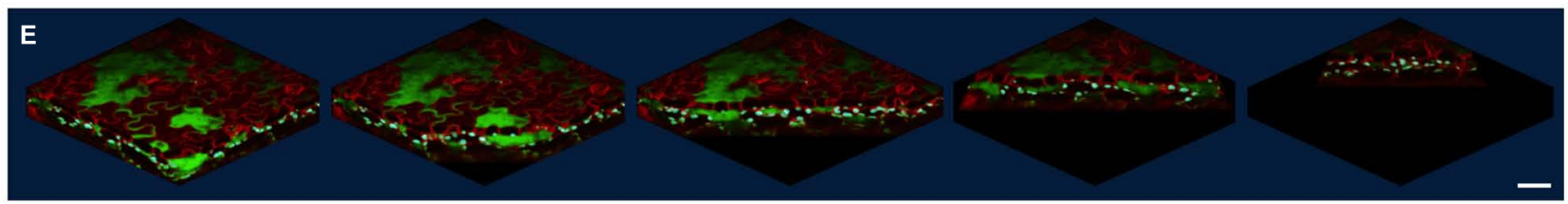
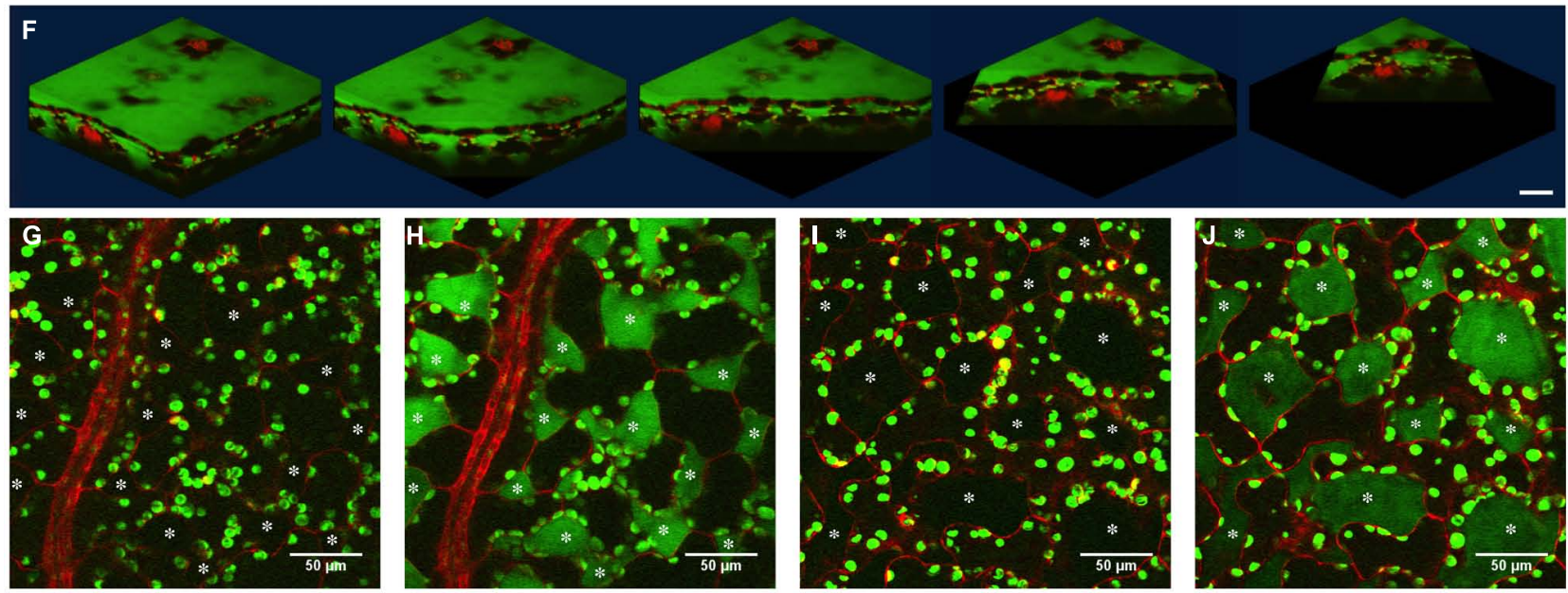

FIGURE 4 | Stimulated Raman scattering imaging of perfluorocarbons in vivo. (A-D) Raman spectra taken at $785 \mathrm{~nm}$, comparing spectra of PFD and (A) PP11, (B) Beta 1-3 glucan, (C) cellulose, and (D) hexane extracted Arabidopsis leaf wax. Peaks labeled one and two were used to tune imaging to $\mathrm{C}-\mathrm{F}$ and $\mathrm{C}-\mathrm{H}$ bonds respectively. (E,F) SRS images representing

Z-stacks taken through Arabidopsis leaves mounted in (E) PFD and (F) PP11. $(\mathbf{G}, \mathbf{H})$ show respectively, off and on resonance images of an Arabidopsis leaf mounted in PP11. (I,J) show respectively, off and on resonance images of an Arabidopsis leaf mounted in PFD. Scale bars are $50 \mu \mathrm{m}$. Asterisks denote airspaces. 
from the stokes beam (1064 $\mathrm{nm})$. As the absorption spectrum of the chloroplasts is so broad, tuning the wavelength of the excitation laser does not affect the strength of the chloroplast signal.

Some of the chloroplasts may appear to be on the extracellular side of the red lines (cell walls) delineating the cells in the images.

This is due to the strong out-of-focus signal from chloroplasts; normally 2-photon excitation is constricted to a small, defined volume and therefore no pinhole is used to filter out-of-focus information. However, as explained, the absorption of the chloroplasts is so strong that where the chloroplast signal overlaps with that of the PFC, this is most likely an imaging artifact due to the strength of the TPA signal from the chloroplasts compared to the red fluorescence in the cells and the SRS from PFC, particularly as the phenomenon is seen in both on and off images. As the Chloroplast signal is very intense signal from adjacent image frames could be leaked into selected image frame (resolution in $\mathrm{z}$ direction is slightly worse than $1 \mathrm{um}$ which is the image step size here). This has the effect of making the chloroplasts appear to be the "wrong" side of the red cell walls.

We cannot categorically exclude the possibility that PFC have penetrated the cells, but consider it to be unlikely as otherwise the TPS from the PFC would be much more ubiquitously distributed.

\section{DISCUSSION}

In this investigation, we show that mounting living leaves in the PFCs, PFD, and perfluoroperhydrophenanthrene (PP11) quantifiably improves the clarity and consistency of images acquired from the mesophyll, for a number of laser-based microscopy techniques including LSCM, 2-photon microscopy, SHG, and SRS.

PFD and PP11 are non-toxic and possess a great carrying capacity for $\mathrm{O}_{2}$ and $\mathrm{CO}_{2}$. PFCs are not miscible with aqueous solutions, which are a disadvantage when trying to deliver bioactive compounds to cells in the leaf, however, this does suggest that PFCs may be of great use as they will not dilute metabolites or signaling molecules present in the cell wall, which could be easily perturbed by aqueous mounting media.

Both PFCs used in this investigation have low surface tensions (19.3 dyne $\mathrm{cm}^{-1}$ for PFD and 21.6 dyne $\mathrm{cm}^{-1}$ for PP11 compared to 72.8 dyne $\mathrm{cm}^{-1}$ for $\mathrm{H}_{2} \mathrm{O}$; Sargent and Seffl, 1970) that are lower than the 25-30 dyne $\mathrm{cm}^{-1}$ required to passively overcome the stomatal barrier (Schönherr and Bukovac, 1972) and readily infiltrate the apoplastic space. This infiltration smooths the optical phase transitions within the mesophyll, resulting in reduced noise and quantifiably clearer images. Moreover, the properties that PFD has displayed for mesophyll - easy infiltration into the tissue, significant improvement in $z$-plane resolution and nontoxicity - may be exploited for more general, in vivo imaging of air-filled or heavily vascularised animal tissues, such as insect spiracles or vertebrate lung, where gaseous exchange is also important and which are primary target for microbial infection. Similarly, PFD may be used advantageously for the perfusion and imaging within organ cavities.

The improvement in image clarity obtained by mounting samples in PP11 is greater than for PFD, which we ascribe to a closer matching of the refractive index of PP11 (1.334) with that of leaf tissue, which has been estimated as 1.4-1.6 depending on wavelength of incident light (Paillotin et al., 1998; Feret et al., 2008) compared to that of PFD (1.313). This was shown by autocorrelation analysis of pixel intensities in LSCM images. This analysis also demonstrated that the use of PP11 is particularly advantageous for imaging shorter wavelengths (i.e., the "green" channel; Figure 3). Increased fluorescence transmission of shorter wavelengths may have important advantages when more than one fluorophore is imaged simultaneously, for example in analyses that require Forster resonance energy transfer (FRET) or co-localisation.

We anticipate that the general improvement in image quality conferred by mounting in PFC media could be further enhanced through an even closer match between the refractive indices of living cells and the PFC mounting medium. 2,2' -thiodiethanol (TDE) has previously been used in this fashion (Staudt et al., 2007); in aqueous solution at varying concentrations, TDE provides a suite of colorless mounting media with tuned refractive indices from 1.33 to 1.52 . Although TDE is not compatible with living samples and used preferentially with fixed specimens, it raises the possibility of a tuneable mounting system composed of two PFCs, each with refractive indices that bracket those of living specimens. These complementary PFCs may then be combined in set proportions to make a mounting medium in which the refractive index is matched to that of any sample.

The presence of C-F bonds in PFCs can be readily distinguished from the $\mathrm{C}-\mathrm{H}$ bonds found in biological molecules. Consequently, PFCs does not impair the imaging of biological molecules by label-free imaging techniques such as CARS microscopy and SRS microscopy (Mansfield et al., 2013). The C-F bond itself can be exploited to visualize the distribution of PFC in biological material. In this investigation, we have exploited this distinction to generate contrast images of the "negative space" within the mesophyll. Images of the apoplast throughout development will enable a better understanding of leaf expansion and growth. Moreover, is will be possible to use such techniques to develop refined models of the airspaces within leaves, and better understand the constraints of gaseous fluxes within.

\section{CONCLUSION}

We have shown that mounting living leaves in PFD and perfluoroperhydrophenanthrene (PP11) improves image resolution for a number of laser-based microscopy techniques including LSCM, TPF, SHG, and SRS microscopy. These compounds allow greater $z$-axis penetration, resulting in clearer micrographs. In addition, PFCs may be used as label-free contrast agents to image the internal architecture of leaves, and enable a more precise understanding of the structural changes that occur during leaf development. As the mesophyll is a primary target for pathogenesis in plants, this technique may also be used to observe the processes of infection deep within the mesophyll. More generally, the use of PFCs as mounting media may be applied to other tissues rich in airspaces, such as animal lungs.

\section{AUTHOR CONTRIBUTIONS}

George R. Littlejohn, Mark D. Fricker, Murray R. Grant, Nicholas Smirnoff, Julian Moger, Jacqueline T. Christmas, Richard M. Everson, and John Love designed the research; George R. Littlejohn, 
Eleanor Witterick, Jessica C. Mansfield, and Julian Moger performed the experiments; George R. Littlejohn, Eleanor Witterick, Jessica C. Mansfield, Jacqueline T. Christmas, Julian Moger, and John Love analyzed the data; George R. Littlejohn, Jessica C. Mansfield, Jacqueline T. Christmas and Jessica C. Mansfield and John Love wrote the manuscript.

\section{ACKNOWLEDGMENTS}

The authors would like to thank John Mansfield and Dawn Arnold for providing GFP-labeled Pseudomonas. Funding for this research was from the Biotechnology and Biological Sciences Research Council (BBSRC), UK. We also acknowledge the Exeter Imaging Network.

\section{REFERENCES}

Brown, R. M., Millard, A. C., and Campagnola P. J. (2003). Macromolecular structure of cellulose studied by second-harmonic generation imaging microscopy. Opt. Lett. 28, 2207-2209. doi: 10.1364/OL.28.002207

Carrión, C. A., Costa, M. L., Martínez, D. E., Mohr, C., Humbeck, K., and Guiamet, J. J. (2013). In vivo inhibition of cysteine proteases provides evidence for the involvement of "senescence-associated vacuoles" in chloroplast protein degradation during dark-induced senescence of tobacco leaves. J. Exp. Bot. 64, 4967-4980. doi: $10.1093 / \mathrm{jxb} / \mathrm{ert} 285$

Cheng, P.-C. (2006). "Interaction of light with botanical specimens," in Handbook of Biological Confocal Microscopy, 3rd Edn, ed. J. P. Pawley (New York: Springer Science+Business Media, LLC), 414-441. doi: 10.1007/978-0-387-455 24-2_21

Cox, G., Moreno, N., and Feijó, J. (2005). Second-harmonic imaging of plant polysaccharides. J. Biomed. Opt. 10:024013. doi: 10.1117/1.1896005

Diaspro, A., and Sheppard, C. J. R. (2002). "Two-photon excitation flouresence microscopy," in Confocal and Two-Photon Microscopy: Foundations, Applications and Advances, ed. A. Diaspro (New York, NY: Wiley-Liss Inc.), 39-73.

Feijó, J. A., and Moreno, N. (2004). Imaging plant cells by two-photon excitation. Protoplasma 223, 1-32. doi: 10.1007/s00709-003-0026-2

Feret, J.-B., François, C., Asner, G. P., Gitelson, A. A., Martin, R. E., Bidel, L. P. R., et al. (2008). PROSPECT-4 and 5: advances in the leaf optical properties model separating photosynthetic pigments. Remote Sens. Environ. 112, 3030-3043. doi: 10.1016/j.rse.2008.02.012

Freudiger, C. W., Min, W., Saar, B. G., Lu, S., Holtom, G. R., He, C. W., et al. (2008). Label-free biomedical imaging with high sensitivity by stimulated raman scattering microscopy. Science 322, 1857-1861. doi: 10.1126/science.1165758

Gest, N., Garchery, C., Gautier, H., Jiménez, A., and Stevens, R. (2013). Light-dependent regulation of ascorbate in tomato by a monodehydroascorbate reductase localized in peroxisomes and the cytosol. Plant Biotechnol. J. 11, 344-354. doi: 10.1111/pbi.12020

Haralick, R. M., Shanmugam, K., and Dinstein, I. (1973). Textural features for image classification. IEEE Trans. Syst. Man Cybern. Vol. SMC-3, No. 6, 610-621. doi: 10.1109/TSMC.1973.4309314

Hoepflinger, M. C., Geretschlaeger, A., Sommer, A., Hoeftberger, M., Nishiyama, T., Sakayama, H., et al. (2013). Molecular and biochemical analysis of the first ARA6 homologue, a RAB5 GTPase, from green algae. J. Exp. Bot. 64, 5553-5568. doi: $10.1093 /$ jxb/ert322

Hutt, H., Everson, R., Grant, M., Love, J., and Littlejohn, G. R., (2013). "How clumpy is my image? using citizen science to evaluate crowdsourced annotation tasks," in Proceedings of the 13th UK Workshop on Computational Intelligence, Guildford, 136-143, ISBN 978-1-4799-1568-2 (http://clumpy.ex.ac.uk/static/paper.pdf)

Inoue, S. (2006). "Foundations of confocal scanned imaging in light microscopy," in Handbook of Biological Confocal Microscopy, 3rd Edn, ed. J. P. Pawley (New York: Springer Science+Business Media, LLC), 1-16.

Johnson, C., Ravichadran, A., Smith, A., Vitha, S., and Holzenburg, A. (2011). Anchoring of FtsZ assemblies to the chloroplast envelope: the role of ARC6. Microsc. Microanal. 17(Suppl. 2), 330-331. doi: 10.1017/S14319276110 02522

Knapp, E., Flores, R., Scheiblin, D., Modla, S., Czymmek, K., and Yusibov, V. (2012). Materials and methods Plasmid DNA. Biotechniques 52, 31-37.
Lambertsen, L., Sternberg, C., and Molin, S. (2004). Mini-Tn7 transposons for site-specific tagging of bacteria with fluorescent proteins. Environ. Microbiol. 6, 726-732. doi: 10.1111/j.1462-2920.2004.00605.x

Littlejohn, G. R., Gouveia, J. D., Edner, C., Smirnoff, N., and Love, J. (2010). Perfluorodecalin enhances in vivo confocal microscopy resolution of Arabidopsis thaliana mesophyll. New Phytol. 186, 1018-1025. doi: 10.1111/j.1469-8137.2010.03244.x

Littlejohn, G. R., and Love, J. (2012). A simple method for confocal imaging of Arabidopsis leaves with perfluorodecalin as infiltrative imaging medium. J. Vis. Exp. 16, pii: 3394. doi: 10.3791/3394

Mansfield, J., Littlejohn, G., Seymour, M., Lind, R., Perfect, S., and Moger, J. (2013). Label-free chemically specific imaging in-planta with stimulated Raman scattering microscopy. Anal. Chem. 85, 5055-5063. doi: 10.1021/ac400266a

Mizutani, G., Sonoda, Y., Sano, H., Sakamoto, M., Takahashi, T., and Ushioda, S. (2000). Detection of starch granules in a living plant by optical second harmonic microscopy. J. Lumin. 87-89, 824-826. doi: 10.1016/S0022-2313(99) 00428-7

Moger, J., Garrett, N. L., Begley, D., Mihoreanu, L., Lalatsa, A., Lozano, M. V., et al. (2012). Imaging cortical vasculature with stimulated Raman scattering and twophoton photothermal lensing microscopy. J. Raman Spectrosc. 43, 668-674. doi: 10.1002/jrs.3156

Nagai, T., Ibata, K., Park, E. S., Kubota, M., Mikoshiba, K., and Miyawaki, A. (2002). A variant of yellow fluorescent protein with fast and efficient maturation for cell-biological applications. Nat. Biotechnol. 20, 87-90. doi: 10.1038/nbt0 102-87

Nandakumar, P., Kovalev, A., and Volkmer, A. (2009). Vibrational imaging based on stimulated Raman scattering microscopy. New J. Phys. 11:033026. doi: 10.1088/1367-2630/11/3/033026

Paillotin, G., Leibl, W., Gapiñski, J., Breton, J., and Dobek, A. (1998). Light gradients in spherical photosynthetic vesicles. Biophys. J. 75, 124-133 doi: 10.1016/S00063495(98)77500-9

Sargent, J. W., and Seffl, R. J. (1970). Properties of perfluorinated liquids. Fed. Proc. 29, 1699-1703.

Schönherr, J., and Bukovac, M. J. (1972). Penetration of stomata by liquids. Plant Physiol. 49, 813-819. doi: 10.1104/pp.49.5.813

Shaw, S. L., and Ehrhardt, D. W. (2013). Smaller, faster, brighter: advances in optical imaging of living plant cells. Annu. Rev. Plant Biol. 64, 351-375. doi: 10.1146/annurev-arplant-042110-103843

Staudt, T., Lang, M. C., Medda, R., Engelhardt, J., and Hell, S. W. (2007). 2,2'thiodiethanol: a new water soluble mounting medium for high resolution optical microscopy. Microsc. Res. Tech. 70, 1-9. doi: 10.1002/jemt.20396

Tschiersch, H., Liebsch, G., Borisjuk, L., Stangelmayer, A., and Rolletschek, H. (2012). An imaging method for oxygen distribution, respiration and photosynthesis at a microscopic level of resolution. New Phytol. 196, 926-936. doi: 10.1111/j.1469-8137.2012.04295.x

Wright, K. M., Chapman, S., McGeachy, K., Humphris, S., Campbell, E., Toth, I. K., et al. (2013). The endophytic lifestyle of Escherichia coli O157: H7: quantification and internal localization in roots. Phytopathology 103, 333-340. doi: 10.1094/PHYTO-08-12-0209-FI

Conflict of Interest Statement: The authors declare that the research was conducted in the absence of any commercial or financial relationships that could be construed as a potential conflict of interest.

Received: 21 December 2013; accepted: 24 March 2014; published online: 23 April 2014.

Citation: Littlejohn GR, Mansfield JC, Christmas JT, Witterick E, Fricker MD, Grant MR, Smirnoff N, Everson RM, Moger J and Love J (2014) An update: improvements in imaging perfluorocarbon-mounted plant leaves with implications for studies of plant pathology, physiology, development and cell biology. Front. Plant Sci. 5:140. doi: 10.3389/fpls.2014.00140

This article was submitted to Plant Cell Biology, a section of the journal Frontiers in Plant Science.

Copyright (ㅇ 2014 Littlejohn, Mansfield, Christmas, Witterick, Fricker, Grant, Smirnoff, Everson, Moger and Love. This is an open-access article distributed under the terms of the Creative Commons Attribution License (CC BY). The use, distribution or reproduction in other forums is permitted, provided the original author(s) or licensor are credited and that the original publication in this journal is cited, in accordance with accepted academic practice. No use, distribution or reproduction is permitted which does not comply with these terms. 